\title{
Decisão em Condições de Incerteza Meteorológica e Proteção de Infraestruturas no Centro de Lançamento de Alcântara
}

\author{
Amaury Caruzzo ${ }^{1}$, Mischel Carmen Neyra Belderrain ${ }^{2}$, Gilberto Fisch ${ }^{3}$, George Young ${ }^{4}$, \\ Christopher Hanlon ${ }^{4}$, Johannes Verlinde ${ }^{4}$ \\ ${ }^{I}$ Department of Atmospheric \& Oceanic Sciences, McGill University, Montreal, QC, Canada. \\ ${ }^{2}$ Departamento de Gestão e Apoio à Decisão, Instituto Tecnológico de Aeronáutica, \\ São José dos Campos, SP, Brasil. \\ ${ }^{3}$ Departamento de Ciências Atmosféricas, Instituto de Aeronáutica e Espaço, \\ São José dos Campos, SP, Brasil. \\ ${ }^{4}$ Department of Meteorology and Atmospheric Science, Pennsylvania State University, \\ University Park, PA, United States of America.
}

Recebido em 15 de Abril de 2016 - Aceito em 25 de Julho de 2016

\begin{abstract}
Resumo
A tomada de decisão utilizando informações meteorológicas muitas vezes é um processo complexo, de alto impacto e, em algumas situações, pode comprometer o funcionamento de instalações tecnológicas. Neste contexto, o objetivo deste artigo é apresentar uma abordagem de apoio à decisão em condições de incerteza meteorológica para a proteção das infraestruturas no Centro de Lançamento de Alcântara. A partir de uma demanda operacional, foi desenvolvido um índice meteorológico de modo a identificar a atitude dos decisores em relação às três características da informação meteorológica: a) probabilidade da previsão de tempo; b) prazo de validade; c) valor da variável atmosférica. Como estudo de caso, foi analisado um problema real de decisão para proteção das instalações do principal centro espacial brasileiro. Através da interação com os usuários na construção do índice, foi possível concluir que para probabilidades baixas $(<20 \%)$ ou para prazos de validade estendidos $(>24 \mathrm{~h})$, o grupo de usuários entrevistados não utiliza a informação meteorológica para tomar decisões, mesmo com o prognóstico de condições extremas. Os resultados quantitativos demonstraram que o modelo de apoio à decisão proposto conseguiu incorporar a atitude do usuário não-meteorologista em relação à incerteza da informação meteorológica.
\end{abstract}

Palavras-chave: índice de decisão meteorológico, risco meteorológico, análise multicritério, centro de lançamento de foguetes, proteção de infraestrutura crítica.

\section{Decision Under Weather Uncertainty and Infrastructure Protection for Alcantara Launch Center}

\begin{abstract}
Decision-making using weather information is often a complex and high impact process, and in some situations, can disturb the functioning of technological facilities. In this sense, the aim of this paper is to present a decision support approach for infrastructure protection under weather uncertainty at the Alcântara Launch Center, in Brazil. From an operational demand, a weather index was developed to identify the decision maker's attitude about the three characteristics of meteorological information: a) weather forecast probability; b) lead time of the information; c) value of a meteorological variable. As a case study, we analyzed a real decision-making problem for the protection of an aerospace vehicle and integration tower in the Brazilian space center. Through the development of an index with the users, it was concluded that for low probabilities $(<20 \%)$ or extended lead time $(>24 \mathrm{~h})$, the group of users do not use weather information to make decisions, even with a prognosis of extreme conditions. The quantitative results showed that the decision model managed to incorporate the attitude of non-meteorologist users regarding the handling of uncertainty in weather forecasts.
\end{abstract}

Keywords: weather decision index, weather risk, multi-criteria analysis, rocket launch center, critical infrastructure protection.

Autor de correspondência: Amaury Caruzzo, amaury.caruzzo@mail.mcgill.ca, acaruzzo@gmail.com. 


\section{Introdução}

A tomada de decisão utilizando informações meteorológicas sempre foi um desafio para a Sociedade. Desde decisões simples até as mais complexas, tais como a evacuação de áreas ameaçadas por furações ou na proteção de infraestruturas, os decisores buscam interpretar a previsão de tempo de forma a estruturar o processo decisório (Oh et al., 2010 e Demuth et al., 2012).

Apesar do desenvolvimento dos sistemas computacionais e de novas técnicas de observação da atmosfera, o prognóstico das condições atmosféricas ainda possuem incertezas (Neal et al., 2014). Além disso, muitas vezes os usuários não-meteorologistas tem o desafio de avaliar a previsão de tempo e tomar decisões baseados em uma informação determinística, sem uma análise das probabilidades. Entretanto Joslyn e Leclerc (2012) e Fan et al. $(2015,2016)$ apresentam que, com a previsão determinística o usuário não é capaz de avaliar completamente o contexto decisório relacionado a incerteza do prognóstico ambiental. Ou seja, a ausência de uma informação probabilística não é adequada quando as preferências dos usuários são dinâmicas e mudam conforme a variação desta incerteza. Por exemplo, a previsão de chuva forte, com prazo de validade de 24 h e $70 \%$ de probabilidade, possui uma expectativa de recompensa diferente em relação à mesma previsão, mas com $20 \%$ de probabilidade (Joslyn e Leclerc, 2013).

Por outro lado, com o uso de técnicas estatísticas é possível estabelecer uma Previsão de Tempo Probabilística (PTP), na qual o valor de cada variável meteorológica está associado a um prazo de validade e a uma probabilidade (Hanlon et al., 2014 e Neal et al., 2014). Com a PTP, os meteorologistas e usuários podem avaliar as possíveis consequências e os respectivos níveis de confiança em relação à previsão de tempo (Caruzzo et al., 2015a). Ainda como apresentado por Slingo e Palmer (2011), do ponto de vista dos usuários, a PTP permite identificar o perfil de risco, as preferências em relação às probabilidades e consequentemente, estimar os impactos no caso de condições meteorológicas desfavoráveis.

$\mathrm{Na}$ abordagem clássica de decisão sob incerteza meteorológica, é estabelecido ' $n$ ' estados da natureza $(\theta)$ com ' $m$ ' alternativas ou ações. Por exemplo, a ocorrência (ou não) de chuva e levar (ou não) um guarda-chuva. A partir da matriz ' $M$ ' é construída uma distribuição de probabilidade de $\theta$ e identificada as preferências dos envolvidos em relação às recompensas da decisão. Considerando que a informação meteorológica possui diversas variáveis, o problema de decisão usando a PTP pode ser modelado como de múltiplos critérios, demandando assim uma aplicação de um método de Análise de Decisão por Multicritérios (Campello-de-Souza, 2007 e Almeida, 2011). De acordo com Keeney; Raiffa (1993) na abordagem multicritério, cada alternativa $a \in A$ (onde $A$ pode ser discreto ou contínuo) está relacionada as consequências multidimensionais de cada critério ' $x$ '. O conjunto dos critérios $X_{j}$ onde $j=(1,2,3, \ldots, n)$, está associada a alternativa ' $a$ ' em um ponto do espaço $n$-dimensional de consequências. Portanto o contexto de decisão é uma seleção de alternativa que promove uma maior satisfação do vetor resultante $X_{1}(a)$, $X_{2}(a), \ldots, X_{n}(a)$ para o usuário. Por outro lado, em uma decisão por multicritérios, dificilmente o resultado esperado maximizará em todas as variáveis meteorológicas, sendo na verdade uma combinação de acordo com a modelagem de preferências do usuário.

Diversos autores buscaram integrar o prognóstico das condições ambientais com métodos consagrados de Análise de Decisão (Clemen, 1997 e Li et al., 2009 e Quiring et al., 2014). Outros artigos também apresentam metodologias para modelar uma decisão sob incerteza meteorológica (Hiramatsu et al., 2007; Joslyn et al., 2011 e Hanlon et al., 2014). Entretanto, como debatido por Schad et al. (2012) e Dolif et al. (2013), o desafio está em desenvolver um processo capaz de representar as interpretações dos diferentes atores em relação ao processo decisório utilizando informações ambientais. Aplicado aos prognósticos meteorológicos, Regnier (2008) e Joslyn e Leclerc (2013) debatem que as variações do comportamento probabilístico nos diferentes prazos de validade não permitem que sejam estabelecidas relações de preferências diretas dos usuários, pois as percepções das consequências também são dinâmicas. Nestas condições, os métodos tradicionais de decisão sob incerteza se tornam inviáveis, pois haveria a necessidade de realizar julgamentos constantes, com prazos e probabilidades diferentes, de forma a avaliar as melhores alternativas a cada novo intervalo de tempo da PTP. Como também indicado por Caruzzo et al. (2015a), a decisão sob incerteza meteorológica requer uma abordagem específica para o uso da previsão de tempo probabilística.

No contexto do Programa Espacial Brasileiro (PEB) o Centro de Lançamento de Alcântara (CLA) no Estado do Maranhão, realiza operações de lançamento de veículos aeroespaciais (Marques e Fisch, 2005 e Silva e Fisch, 2014). Durante estas operações, a informação meteorológica é relevante não somente para determinar a janela exata de lançamento do foguete, como também para a proteção de todas as instalações do centro espacial (Vaughan e Johnson, 2013). Guikema et al. (2010) também destaca que, com eventos extremos é necessário identificar as melhores alternativas para a redução do risco meteorológico e a respectiva segurança das infraestruturas.

Assim o objetivo deste trabalho é apresentar uma abordagem de apoio à decisão em condições de incerteza meteorológica para a proteção das infraestruturas do CLA, considerando usuários não-meteorologistas. Foi desenvolvido um índice denominado Índice de Decisão Meteorológico (IDM) de modo a identificar a atitude (preferências) dos usuários em relação às características da previsão de tempo. No caso de eventos meteorológicos adversos, ações 
de mitigação devem ser tomadas no CLA para evitar danos e potenciais prejuízos durante a missão espacial. Como estudo de caso, foi utilizado um problema de decisão operacional para a proteção do Veículo Lançador de Satélite (VLS) e da Torre Móvel de Integração (TMI) no CLA, detalhado a seguir.

\section{Materiais e Métodos}

Como debatido por Wilson e Giles (2013) e Caruzzo et al. (2015) existem desafios em transformar a informação meteorológica em uma decisão operacional, principalmente para usuários que não possuem conhecimento técnico em ciências atmosféricas ou de como é elaborada uma previsão de tempo probabilística. Neste sentido, o desenvolvimento do Índice de Decisão Meteorológico (IDM) foi motivado a partir de uma demanda de apoio ao processo decisório nas operações de lançamento de foguetes no CLA, mais especificamente na proteção do foguete VLS e TMI em caso de condições meteorológicas adversas. O VLS é integrado internamente na TMI e no momento que antecede ao lançamento, a TMI recua e o VLS fica exposto às condições ambientais de tempo presente, como apresentado na Fig. 1.

A construção do modelo de decisão e do IDM foi dividida em quatro etapas, sendo: a) Estruturação do Problema; b) Construção do índice de decisão, c) Desenvolvimento do modelo de apoio à decisão, d) Resultados e recomendações.

\subsection{Etapa 1: Estruturação do problema}

Esta etapa tem como finalidade compreender o problema de decisão utilizando a previsão de tempo no contexto das missões de lançamento de foguete. Foram entrevistados um total de vinte e três usuários, entre meteorologistas, atores e decisores da alta administração do Programa Espacial Brasileiro. Para analisar as entrevistas foi aplicado o método de Estruturação de Problema denominado de Strategic Options Development and Analysis (SODA) (Ackermann e Eden, 2001 e Georgiou, 2010). Com o método

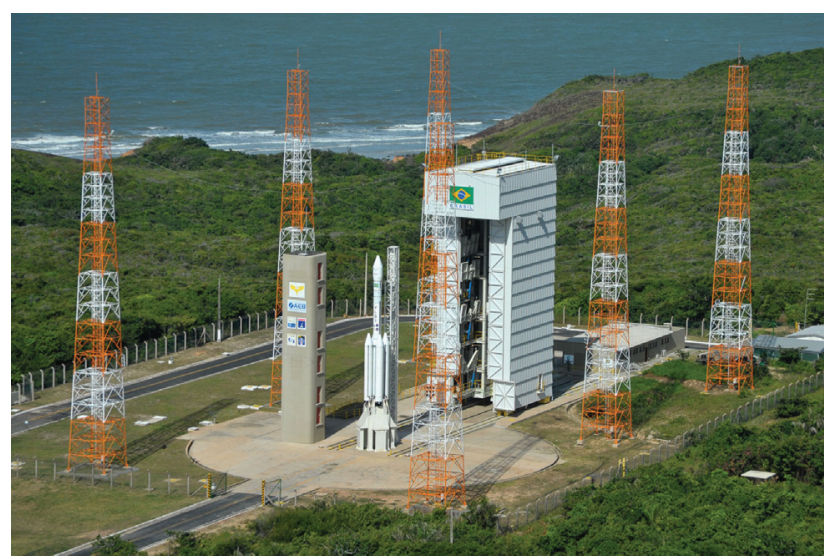

Figura 1 - Foguete VLS-1 próximo a TMI. (fonte: Operação Salinas/CLA).
SODA foram construídos mapas individuais e agregados com os diversos conceitos dos entrevistados. Conforme apresentado por Caruzzo et al. (2015), através da interação com os diversos atores e com o uso das técnicas do método SODA, foi possível identificar as preferências, os valores, julgamentos e os perfis dos usuários em relação as variáveis atmosféricas e a PTP. A partir destas percepções, também foram identificadas as vulnerabilidades, os riscos meteorológicos, os critérios e os limites operacionais do VLS e da TMI.

Alguns valores de vulnerabilidade e limites operacionais das infraestruturas do CLA foram estabelecidos durante a definição de requisitos da construção. Os demais valores foram obtidos por consenso entre os usuários entrevistados e são denominados como Limites Meteorológicos (LM), classificados em três níveis:

- Limite Meteorológico-1 ( $\left(\mathrm{LM}_{1}\right)$ : considerado o valor da variável (máximo) para a plena operação do VLS ou TMI, sem nenhum tipo de restrição ambiental (será o nível 'melhor viável').

- Limite Meteorológico-2 $\left(\mathrm{LM}_{2}\right)$ : é o valor máximo em que o VLS ou TMI pode suportar sem ocorrer nenhum tipo de dano estrutural devido a condições meteorológicas extremas (será o nível 'pior aceitável'). O $\mathrm{LM}_{2}$ é o valor a partir que a infraestrutura pode ser fisicamente comprometida, tais como ventos de grande intensidade, por exemplo.

- Limite Meteorológico intermediário $\left(\mathrm{LM}^{*}\right)$ : para a classificação dos riscos meteorológicos, foi definido um valor intermediário entre os dois limites colocados anteriormente, que detalhado a seguir.

Para o estudo de caso, foram estabelecidas quatro variáveis meteorológicas relevantes na proteção do foguete VLS e da TMI, a saber: taxa de precipitação (chuva), velocidade do vento em superfície, distância na ocorrência de relâmpagos e o valor do campo elétrico atmosférico no centro espacial (que pode induzir a ocorrência de descargas atmosféricas). Vale destacar que as variáveis meteorológicas serão os critérios do modelo de decisão. Na Tabela 1, são apresentados os LMs para cada critério.

\subsection{Etapa 2: Índice de decisão}

Nesta etapa foi desenvolvida uma abordagem quantitativa para transformar os valores da informação meteorológica (observação + previsão) em um índice de decisão único. Na literatura, índices têm aplicações em diversos contextos, seja na análise de riscos (MacKenzie, 2014) ou para alertas meteorológicos (Wilson e Giles, 2013). Ainda como destacado por MacKenzie (2014), o desenvolvimento de um novo índice deve ser adequado ao público-alvo, a fim de determinar o formato final e a respectiva aplicação. Como debatido por Neal et al. (2014) e Caruzzo et al. (2015), para interpretar a previsão de tempo e transformá-la em um índice de decisão, é necessário incorporar as percepções dos usuários não-meteorologistas em relação às três 
Tabela 1 - Limites meteorológicos de cada critério do estudo de caso.

\begin{tabular}{lcccc}
\hline Critério & Unidade & $\mathrm{LM}_{1}$ & $\mathrm{LM}^{*}$ & $\mathrm{LM}_{2}$ \\
\hline $\begin{array}{l}\text { Taxa de precipitação } \\
\text { (chuva) }\end{array}$ & $\mathrm{mm} \mathrm{h}^{-1}$ & 1 & 10,5 & 20 \\
$\begin{array}{l}\text { Velocidade do vento em } \\
\text { superfície }\end{array}$ & $\mathrm{m} \mathrm{s}^{-1}$ & 10 & 25 & 40 \\
$\begin{array}{l}\text { Ocorrência de relâmpago } \\
\text { distância) }\end{array}$ & $\mathrm{km}$ & 50 & 30 & 10 \\
$\begin{array}{l}\text { Valor do campo elétrico } \\
\mathrm{kV} \mathrm{m}^{-1}\end{array}$ & 0,6 & 0,9 & 1,2 \\
\hline
\end{tabular}

Fonte: entrevistas.

características específicas da informação meteorológica, sendo:

- Probabilidade da informação meteorológica (\%)

- Prazo de validade da previsão de tempo (horas, dias)

- Valor da variável meteorológica considerada (vento, chuva, entre outras)

Como apresentado anteriormente, a decisão utilizando a informação meteorológica pode ser caracterizada como um problema de múltiplos critérios. Nos métodos multicritérios, Montibeller e Franco (2007) e Ensslin et al. (2010) propõem um instrumento para melhorar o entendimento do usuário a construção de níveis de impacto (ou limites) e uma avaliação de desempenho que representa as preferências do usuário por meio da chamada Função de Valor. Esta função é uma forma de quantificar a intensidade das preferências do usuário, pela diferença de atratividade entre os valores. Ou seja, por meio de uma escala numérica são identificados os pontos entre os limites que permitem o ajuste de uma função matemática que represente as preferências do usuário. Nesta pesquisa, todas as Funções de Valor foram determinadas para um perfil único, com a validação do usuário mais sênior. Este processo também é chamado de elicitação de preferências (Campello-de-Souza, 2007 e Almeida, 2011).

Inicialmente foram construídas três conjuntos de funções de valor parciais, uma para cada característica da informação meteorológica, em relação às probabilidades $(p)$, aos prazos de validade $(t)$ e aos critérios meteorológicos $(x)$. A primeira função de valor parcial, representada por $I(p)$, está relacionada à probabilidade da PTP. Como demostrado por Caruzzo et al. (2015), para o grupo de usuários entrevistados do $\mathrm{PEB}$, os valores acima de $85 \%$ na previsão foi considerado como nível melhor [=1], sendo este o primeiro valor ancora do nível de impacto e $20 \%$ foi acatado como nível pior $[=0]$, sendo este o segundo valor ancora da escala. Neste estudo de caso, devido à limitação na interação com os usuários, foi adotada uma função linear entre os dois níveis de impacto de todas as características da informação meteorológica, como exposto na Eq. (1) (para a probabilidade). Entretanto, é importante destacar que como demostrado por Campello-de-Souza (2007) e Brito; Almeida (2012) funções mais complexas como curvas logís- ticas ou exponenciais podem representar muito bem o perfil dos usuários com escalas de $[0,1]$.

$$
I(p)=\left\{\begin{array}{c}
1, p \geq 85 \\
\left(\frac{p}{65}\right) \quad-0,308,20 \leq p<85 \\
0, p<20
\end{array}\right.
$$

A segunda função de valor parcial é em relação ao prazo de validade da informação meteorológica $I(t)$. A PTP tem uma validade que pode variar de poucos minutos (nowcasting) até um período de alguns dias (usualmente máximo de 10 dias). Para os usuários entrevistados, os valores âncoras para os prazos de validade foram: até $2 \mathrm{~h}$, como o nível melhor [1] e acima de 24 h, o nível pior [0] (Eq. (2)).

$$
I(t)=\left\{\begin{array}{r}
1, t \leq 2 \\
-\left(\left(\frac{t}{22}\right)-1,091\right), 2<t \leq 24 \\
0, t>24
\end{array}\right.
$$

Para as funções de valor $I(x)$ foi necessário construir uma equação para cada um dos critérios meteorológicos, que neste estudo são quatro (Tabela 1). Foi estabelecido como condição de contorno que a previsão tenha $p \geq 85 \%$ e $t \leq 2 \mathrm{~h}$, ou seja, $I_{p}=I_{t}=1$ (' $p$ ' e ' $t$ ' no nível 'melhor viável'). O usuário sênior estabeleceu os valores ancoras de cada um dos critérios como os próprios Limites Meteorológicos definidos anteriormente. $\mathrm{O}$ nível melhor foi o $\mathrm{LM}_{1}$ e o nível pior o $\mathrm{LM}_{2}$. O LM* é o valor intermediário, onde a função de valor é $[=0,5]$. As equações que definem as funções de valor das quatro variáveis meteorológicas do estudo de caso são apresentadas no Apêndice 1.

A partir dos LMs para cada critério ' $x$ ' foi estabelecida uma tabela de classificação de perigo meteorológico para a proteção das infraestruturas do CLA (Tabela 2).

A agregação destas três funções de valor (de cada critério) em um índice único é denominado de Índice de Decisão Meteorológico parcial $(i d m)$ e representa uma escala de intensidade de preferência (Ensslin et al., 2001) em relação a cada variável meteorológica. A partir da informação meteorológica, o idm estabelece um valor na escala entre 0 e 1 conforme o perfil do usuário. Para a construção da função $i d m$ foram assumidas algumas premissas iden-

Tabela 2 - Classificação do perigo meteorológico a partir dos valores dos LMs.

\begin{tabular}{lcc}
\hline $\begin{array}{l}\text { Nível de perigo } \\
\text { meteorológico }\end{array}$ & $\begin{array}{c}\text { Valor do critério } \\
\text { (somente relâmpago) }\end{array}$ & $\begin{array}{c}\text { Valor do critério } \\
\text { (demais variáveis) }\end{array}$ \\
\hline Baixo $\left(\theta_{1}\right)$ & $x \geq \mathrm{LM}_{1}$ & $x \leq \mathrm{LM}_{1}$ \\
Médio $\left(\theta_{2}\right)$ & $\mathrm{LM}_{1}>x>\mathrm{LM}^{*}$ & $\mathrm{LM}_{1}<x<\mathrm{LM}^{*}$ \\
Alto $\left(\theta_{3}\right)$ & $\mathrm{LM}^{*} \geq x>\mathrm{LM}_{2}$ & $\mathrm{LM}^{*} \leq x<\mathrm{LM}_{2}$ \\
Extremo $\left(\theta_{4}\right)$ & $x \leq \mathrm{LM}_{2}$ & $x \geq \mathrm{LM}_{2}$ \\
\hline
\end{tabular}


tificadas nas preferências dos usuários entrevistados do PEB (Caruzzo et al., 2015):

- Não existem diferenças de perfil em relação às probabilidades e prazos de validade entre as quatro variáveis meteorológicas;

- Com probabilidades baixas $(\mathrm{p}<20 \%$ ) ou prazo de validade estendido $(t>24 \mathrm{~h})$, o usuário não utiliza a previsão de tempo para tomar decisões, ou seja, os valores de ' $p$ ' e ' $t$ ' penalizam ao máximo a utilidade da informação meteorológica e $i d m$ assume um valor fixo máximo;

- Com probabilidades altas $(p \geq 85 \%)$ e prazo de validade curto $(t \leq 2 \mathrm{~h})$, o usuário utiliza e confia plenamente na informação meteorológica para tomar decisões, ou seja, ' $p$ ' e ' $t$ ' não penalizam a informação meteorológica e o $i d m$ assume o valor da função parcial do critério meteorológico ' $x$ '

- Em casos de eventos severos (risco extremo, $\theta_{4}$ ) com alta probabilidade $(p \geq 85 \%)$ e prazo de validade curto $(t \leq 2 \mathrm{~h})$, o usuário utiliza e confia na informação para tomar decisão e o idm assume um valor mínimo.

A partir destas premissas e da agregação em um valor único, foi possível desenvolver a função Índice de Decisão Meteorológico parcial para cada variável meteorológica ' $x$ ', que melhor representa as preferências do usuário (Eq. (3)):

$$
i d m_{x}=I_{x}+\left(1-I_{x}\right)\left(1-\left(I_{p} I_{t}\right)^{\rho}\right)
$$

em que $i d m_{x}=$ índice de decisão meteorológico para o critério ' $x$ ', $I_{x}=$ função de valor para a variável ' $x$ ' da informação meteorológica, $I_{p}=$ função de valor para a probabilidade ' $p$ ', $I_{t}=$ função de valor para o prazo de validade ' $t$ ' e $\rho=$ parâmetro de ajuste do $i d m$ parcial $[=0,5]$.

O parâmetro ' $\rho$ ' é uma forma de ajustar o valor da função $i d m$ conforme o perfil do usuário. Para isso é importante realizar o procedimento de interação com o usuário de forma adequada, pois a função deve representar consistentemente os julgamentos de valor que o usuário utiliza no processo decisório.

\subsection{1. Índice de Decisão Meteorológico global}

O Índice de Decisão Meteorológico global ou multicritério (IDM) é construído a partir do idm (parcial) de todas variáveis consideradas no modelo. A função $I D M$ global tem como finalidade organizar o conjunto dos critérios meteorológicos relacionados à decisão e auxiliar o usuário na seleção de alternativas no prazo de validade da previsão de tempo. Devido à incerteza das condições meteorológicas futuras que proporciona diversas possibilidades no espaço dimensional de consequências, os potenciais cenários foram determinados pela variação dos quatro níveis de risco das variáveis meteorológicas (Tabela 2).

Estabelecidos os cenários meteorológicos, o usuário demostrou estar disposto a fazer compensações (ponderar) entre as variáveis para identificar a melhor alternativa. Por exemplo: em um cenário onde as combinações estabelecem dois critérios no nível de risco meteorológico alto, para o usuário se os critérios forem chuva e vento ou chuva e relâmpago, são consideradas condições diferentes e, portanto são indicadas ações de mitigação distintas. Logo assumimos como um método multicritério compensatório (Almeida, 2011), sendo necessário estabelecer os pesos entre os critérios meteorológicos selecionados. Estes pesos foram identificados pela abordagem Swing Weights (Montibeller e Franco, 2007 e Gomes et al., 2011). Esta técnica define uma escala numérica associada às preferências entre as variáveis meteorológicas. Isto é, uma forma de ponderar a ordem de importância das variáveis, adotando valores entre 0 até 100 , sendo o valor mais alto, o mais importante. A partir da identificação dos pesos dos critérios para a proteção do VLS e da TMI, foi elaborada a Estrutura Hierárquica de Valor (Fig. 2).

Com um conjunto de informações meteorológicas ' $i$ ' (observação em tempo real + previsão de tempo) a função Índice de Decisão Meteorológica global (IDM) foi construída a partir do conceito da metodologia multicritério e pode ser determinada pela Eq. (4) (Belton e Stewart, 2002 e Gomes et al., 2011).

$$
\operatorname{IDM}(i)=\sum_{j=1}^{n} k_{j} i d m_{j}(i)
$$

em que $i d m_{j}(i)=$ valor do índice de decisão meteorológico parcial de cada critério ' $j$ ' na condição meteorológica ' $i$ ' e $k_{j}=$ peso do critério, sendo $\sum_{j=1}^{n} k_{j}=1$. A função $I D M$ global é uma função de valor aditiva que determina os valores totais de cada condição meteorológica, na qual a opção recomendada para o usuário será a alternativa que obter o resultado numérico de acordo com a classificação das ações de mitigação, apresentada a seguir.

\subsection{Etapa 3: Apoio à decisão, cenários e mitigação}

No estudo de caso, foram definidos quatro cenários de referências a partir dos níveis de perigo meteorológico, além de outros nove cenários intermediários (Tabela 3). É importante mencionar que neste estudo não foram avaliadas as probabilidades de ocorrência de cada um dos cenários, pois o objetivo é apoiar o usuário através de uma escala de classificação de todas as alternativas possíveis.

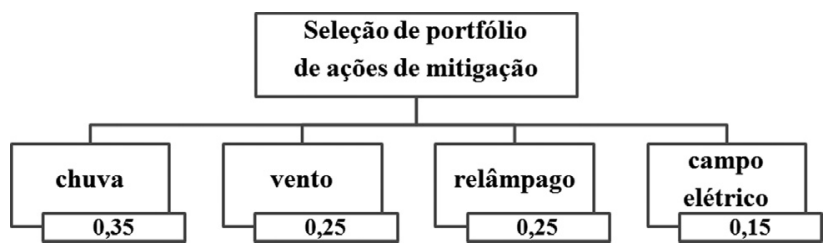

Figura 2 - Hierárquica do modelo de decisão com os respectivos pesos entre as variáveis 
Tabela 3 - Relação dos potenciais cenários meteorológicos e os respectivos valores referência do IDM máximo.

\begin{tabular}{llc}
\hline Cenário & \multicolumn{1}{c}{ Observação } & IDM máximo \\
\hline S1 (risco baixo) & todas as variáveis meteorológicas no nível de perigo baixo (condições totalmente favoráveis) & 1 \\
S2 & 1 variável no nível de perigo médio & 0,9997 \\
S3 & 2 variáveis no nível de perigo médio & 0,9991 \\
S4 & 3 variáveis no nível de perigo médio & 0,9986 \\
S5 (risco médio) & todas as variáveis meteorológicas no nível de perigo médio & 0,9950 \\
S6 & 1 variável no nível de perigo alto & 0,9250 \\
S7 & 2 variáveis no nível de perigo alto & 0,8000 \\
S8 & 3 variáveis no nível de perigo alto & 0,6749 \\
S9 (risco alto) & todas as variáveis meteorológicas no nível de perigo alto & 0,5000 \\
S10 & 1 variável no nível de perigo extremo & 0,8500 \\
S11 & 2 variáveis no nível de perigo extremo & 0,6000 \\
S12 & 3 variáveis no nível de perigo extremo & 0,3499 \\
S13 (risco extremo) & todas as variáveis meteorológicas no nível de perigo extremo (condições de evento extremo) & 0 \\
\hline
\end{tabular}

Obs.: $j=(1, \ldots, 4)$.

Através da variação nos valores das variáveis meteorológicas, foi possível determinar os limites da função $I D M$ mínimos e máximos para cada cenário ' $S$ ' mantendo $I_{p}=I_{t}$ $=1$. Nestas condições, quando o critério estiver no nível de risco mais baixo, o $I D M$ será máximo $(I D M \rightarrow 1)$ e para risco extremo, o $I D M$ será mínimo $(I D M \rightarrow 0)$. Para identificar os limites do IDM foi utilizada a estrutura hierárquica entre os atributos (Fig. 2) e calculado os valores finais do menor para o maior peso. O valor do $I D M$ mais elevado em cada cenário foi assumido como o limite máximo, ou seja, o nível de risco é mais reduzido ponderando todos os critérios meteorológicos (última coluna na Tabela 3). Este valor de referência de cada cenário foi utilizado para definir o IDM limite para o conjunto de alternativas de decisão.

Neste estudo de caso foi estabelecido com o usuário mais sênior que, as alternativas de decisão são grupos de ações de mitigação organizadas de forma a ter um gerenciamento de portfólios. Isto é, o portfólio de mitigação é um conjunto de ações a serem executadas pela equipe operacional do CLA à medida que as condições meteorológicas se deterioram. A classe de portfólio é recomendada com a finalidade de maximizar a proteção das instalações e preservar vidas humanas. Foram definidos um total de seis portfólios no estudo de caso (Tabela 4). A recomendação do portfólio de mitigação ' $M$ ' decore da indicação prévia pelo usuário em relação ao cenário ' $S$ ' (Tabela 3), onde é possível também obter os valores dos IDM limites. Por exemplo, para o cenário $S 9$ o usuário indicou o portfólio $M 5$, portanto o valor do IDM limite será 0,5 , como demostrado na Tabela 4.

De acordo com Vaughan (1996) qualquer programa espacial possui custos altos e riscos elevados, portanto todas as ações para minimizar potenciais acidentes devem ser identificadas e previamente estabelecidas. Neste estudo de caso, foi assumido que todos os portfólios de mitigação podem ser implantados de forma imediata e independente dos custos ou de outras limitações operacionais. Por não ser objeto desta pesquisa, também não foram quantificados os impactos das ações de mitigação na redução dos riscos, pois haveria a necessidade de uma investigação detalhada de todas as vulnerabilidades e dos diversos custos financeiros do centro espacial. Na Fig. 3, é apresentada a estrutura geral do modelo de decisão para a proteção de instalações do CLA utilizando a função IDM para este estudo.

As observações meteorológicas e a PTP são assimiladas pelo modelo de decisão e os valores das funções $i d m$

Tabela 4 - Relação dos portfólios de mitigação de acordo com os cenários e o valor do IDM máximo correspondente.

\begin{tabular}{lccc}
\hline Cenário referência & Classe portfólio & Ações de mitigação & IDM limite \\
\hline S1 & M1 (nenhuma ação) & nenhuma ação de mitigação é executada & $\leq 1,0000$ \\
S3 & M2 & proteção do VLS e da carga útil com a TMI & $\leq 0,9991$ \\
S10 & M3 & suspensão de todas as atividades externas a TMI & $\leq 0,8500$ \\
S8 & M4 & suspensão de todas as atividades internas na TMI & $\leq 0,6749$ \\
S9 & M5 & evacuação do pessoal próximo ao VLS e da TMI & $\leq 0,5000$ \\
S12 & M6 (todas as ações) & suspensão de todas as atividades no CLA (condição de evento & $\leq 0,3499$ \\
& & meteorológico extremo) &
\end{tabular}




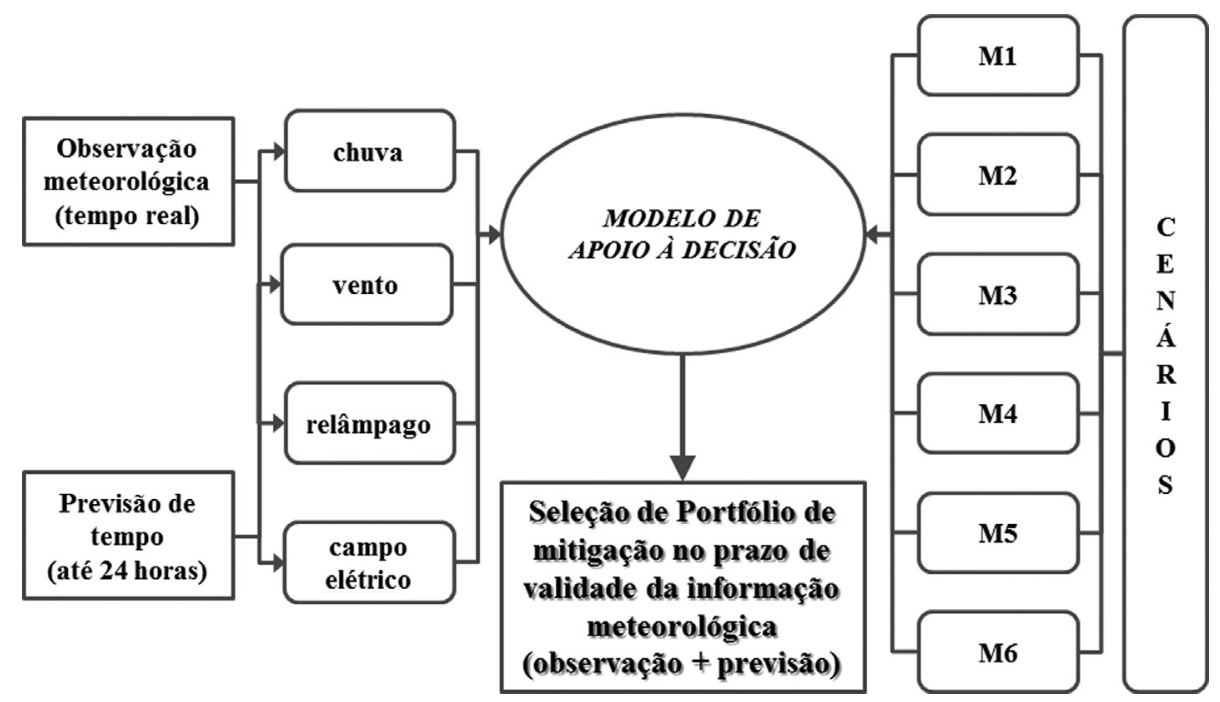

Figura 3 - Proposta do modelo de decisão para o estudo de caso.

(parciais) e $I D M$ (global) para cada prazo de validade da informação meteorológica são calculados. De acordo com os valores do IDM limites, são recomendados os portfólios de mitigação previamente estabelecidos pelo usuário (Tabela 4). Uma vez definidas as funções em relação aos três parâmetros (probabilidade, prazo e valor da variável), as recomendações são atualizadas a cada nova PTP e sem a necessidade de novas interações com o usuário.

\section{Resultados e Discussão}

$\mathrm{Na}$ aplicação do modelo de decisão neste estudo foram definidas três condições meteorológicas hipotéticas para avaliação da proteção do foguete VLS e da TMI (Tabela 5). Foram adotados o prazo máximo de $24 \mathrm{~h}$ e a mesma distribuição de probabilidades para as três categorias. No Apêndice 2 são demostrados os detalhes das condições meteorológicas utilizadas e as etapas nos cálculos dos índices deste estudo de caso.

$\mathrm{Na}$ aplicação deste modelo, é disponibilizado para o usuário o conjunto da informação meteorológica, incluindo a observação em tempo real e a Previsão de Tempo Probabilística, com prognóstico a cada $3 \mathrm{~h}$ e prazo máximo de

Tabela 5 - Características das condições meteorológicas utilizadas no estudo de caso.

\begin{tabular}{|c|c|}
\hline Condição meteorológica & Características \\
\hline Otimista (O) & $\begin{array}{l}\text { as condições iniciam no cenário } \mathrm{S} 2 \text { e no } \\
\text { prazo de } 24 \text { h passam para o cenário } \mathrm{S} 1\end{array}$ \\
\hline Intermediário (M) & $\begin{array}{l}\text { as condições meteorológicas se mantêm no } \\
\text { cenário } \mathrm{S} 4 \text { durante a toda a previsão até o } \\
\text { prazo de } 24 \mathrm{~h}\end{array}$ \\
\hline Pessimista $(\mathrm{P})$ & $\begin{array}{l}\text { as condições iniciam com o cenário S5 e no } \\
\text { prazo de } 24 \text { h passam para o cenário } \\
\text { S11, caracterizando como um evento } \\
\text { meteorológico extremo }\end{array}$ \\
\hline
\end{tabular}

24 h. O problema de decisão consistiu em selecionar portfólios para a proteção do VLS e da TMI em condições meteorológicas desfavoráveis, além de salvaguardar o pessoal técnico que desenvolve atividades de integração e montagem do veículo aeroespacial. Definido todos os parâmetros do modelo de acordo com a Fig. 3, foram calculados os IDM globais para todas as condições hipotéticas, conforme apresentado na Tabela 6 .

O resultado final do modelo de apoio à decisão são os valores do IDM global para cada intervalo no prazo de validade da informação e com a respectiva recomendação de portfólio (Tabela 4). Para o usuário não-meteorologista, um quadro simplificado na apresentação das recomendações facilita a interpretação dos resultados. Operacionalmente, este quadro auxilia também no processo de tomada de decisão com um intervalo de tempo reduzido. Na Fig. 4, é apresentado o comportamento das curvas das funções $I D M$ para as três condições ao longo do prazo de validade da informação meteorológica de $24 \mathrm{~h}$.

A principal característica dos resultados é que para as três condições hipotéticas, o valor do IDM tende a um [1] quando o prazo se aproxima de $24 \mathrm{~h}$. Isto acontece devido às penalidades nas funções $i d m$ parciais, que ocorrem quando as probabilidades ' $p$ ' e os prazos de validade ' $t$ ' não estão no nível 'melhor viável'. Em outras palavras, quando o termo $\left(1-\left(I_{p} I_{t}\right)^{\rho}\right)$ da Eq. (3) é diferente de zero $[\neq 0]$ existe um aumento no valor do $i d m$ devido à probabilidade mais baixa e/ou ao prazo de validade estendido. Ou seja, quando $I_{p}$ e/ou $I_{t} \rightarrow 0$, a função $i d m \rightarrow 1$. Vale destacar que, a distribuição das probabilidades neste estudo de caso é continuamente decrescente (100 para 70\%) no prazo de $24 \mathrm{~h}$ (ver Apêndice 2). Por outro lado, quando este termo na equação é zero $[=0]$ não ocorrem penalidades, pois ' $p$ ' e ' $t$ ' estão no nível 'melhor viável' para o usuário, portanto $I_{p}=I_{t}=1$. 
Tabela 6 - IDMs globais para as três condições meteorológicos do estudo de caso e a respectiva recomendação de portfólio de mitigação em intervalos a cada $3 \mathrm{~h}$.

\begin{tabular}{lccccccccc}
\hline & \multicolumn{8}{c}{ Prazo de validade da informação meteorológica (h) } \\
\cline { 2 - 10 } & 0 & 3 & 6 & 9 & 12 & 15 & 18 & 21 & 1 \\
\hline IDM-O & 0,8833 & 0,9511 & 0,9848 & 1 & 1 & 1 & 1 & 1 & $M 1$ \\
portfólio & $M 2$ & $M 2$ & $M 2$ & $M 1$ & $M 1$ & $M 1$ & $M 1$ & $M 1$ & 0,931 \\
IDM-M & 0,7377 & 0,7960 & 0,8413 & 0,8566 & 0,8607 & 0,8892 & 0,9126 & 0,9383 & 0,9981 \\
portfólio & $M 3$ & $M 3$ & $M 3$ & $M 2$ & $M 2$ & $M 2$ & $M 2$ & $M 2$ & $M 2$ \\
IDM-P & 0,7446 & 0,7234 & 0,6981 & 0,6584 & 0,6275 & 0,6782 & 0,7324 & 0,8250 & 0,9949 \\
portfólio & $M 3$ & $M 3$ & $M 3$ & $M 4$ & $M 4$ & $M 3$ & $M 3$ & $M 3$ & $M 2$ \\
\hline
\end{tabular}

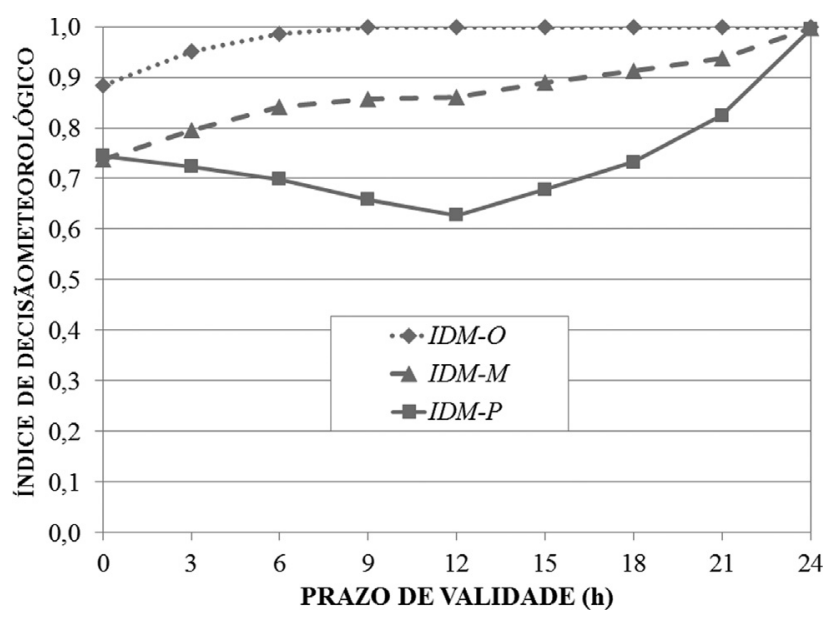

Figura 4 - Função IDM global para as três condições meteorológicas.

Como decorrência direta das penalidades é possível observar que a curva para a condição intermediária (IDM$M)$, apesar dos valores da PTP se manterem no mesmo cenário $\mathrm{S} 4$, a função $I D M$ tem valores crescentes no período. Isso indica que o usuário se torna mais propenso ao risco à medida que os prazos ficam maiores e/ou quando as probabilidades de ocorrência se tornam menores. Podemos afirmar também que, mesmo nas condições meteorológicas extremas $(I D M-P)$ o usuário também se torna mais propenso a risco e, como indicado pelo modelo, deixa de utilizar a PTP para tomar decisões para períodos superiores a $24 \mathrm{~h}$. Após este prazo o $I D M \rightarrow 1$ e de acordo com a Tabela 4 é recomendado o Portfólio M1: "nenhuma ação de mitigação é executada". Ou seja, o usuário aguarda uma atualização da previsão com o prazo mais reduzido antes de selecionar algum portfólio de mitigação. Este julgamento pode ser justificado pelo seguinte comentário de um dos entrevistados: "a previsão de tempo tem uma incerteza muito grande, então eu espero o máximo que puder... até $2 \mathrm{~h}$ antes do lançamento".

Para que a informação meteorológica seja considerada no processo decisório, é preciso avaliar a confiança do usuário na previsão e o respectivo perfil de risco individual. Neste trabalho, isto foi estabelecido através da determi- nação das preferências em relação às probabilidades, aos prazos de validade e aos valores dos critérios atmosféricos. É exatamente esta a inovação do Índice de Decisão Meteorológico: identificar quando a previsão de tempo tem utilidade para o usuário (alta probabilidade e prazo de validade curto) e antecipar a respectiva mudança de atitude quando os valores destas características da informação são alterados. Na Tabela 7, é apresentada uma síntese da atitude do usuário em relação à PTP para este estudo de caso.

Como debatido, existe uma natureza subjetiva no processo decisório utilizando informações meteorológicas. Neste aspecto, podemos avaliar a potencial variabilidade dos IDM parciais e global devido a uma mudança de preferências dos usuários (ou com diferentes decisores) por meio da Análise de Sensibilidade (AS). Inicialmente foram comparados os valores dos $i d m$ parciais dos quatro critérios do modelo original para o cenário S4 do estudo de caso. Neste cenário da AS, três variáveis estão na condição de risco meteorológico médio: chuva $(r)$, vento $(w)$ e campo elétrico $(e f)$. A ocorrência de relâmpagos $(l)$ se mantém no nível de risco baixo, portanto o $i d m_{l}=1$ durante todo o período (Fig. 5). Posteriormente, foram modificados os pesos na função $I D M$ global de cada um dos quatro critérios durante todo o prazo de validade da informação meteorológica e recalculados os valores dos IDM globais. Foram adotados o peso de 0,7 para um critério preferencial do usuário e 0,1 para os demais, fazendo um rodízio entre as quatro variáveis meteorológicas. Os valores dos novos quatro IDM globais da AS e do $I D M-M$ (modelo original) são apresentados na Fig. 6.

Através da AS foram identificadas algumas características nas variabilidades dos IDM (parcial e global). Por exemplo, a curva do $I D M-l$ (Fig. 6) representa o peso 0,7 para a ocorrência de relâmpago e 0,1 para os demais. Nesta condição, o valor do IDM global no prazo de $3 \mathrm{~h}$ é aproximadamente 0,92 . Já para o $I D M-r$ (peso 0,7 para chuva e 0,1 para os demais), o valor do IDM global é reduzido para 0,76 , indicando um portfólio de mitigação diferente (Tabela 4). Por ser um modelo multicritério compensatório, outra característica é que ao longo do prazo de validade da informação meteorológica, a função $I D M$ global tem uma tendência a seguir às curvas do idm parcial do critério de maior 
Tabela 7 - Atitude do usuário em relação às variações nas probabilidades e nos prazos de validade da informação meteorológica.

\begin{tabular}{|c|c|c|c|}
\hline \multicolumn{2}{|c|}{ Condições informação meteorológica } & \multirow[t]{2}{*}{ Atitude do usuário } & \multirow[t]{2}{*}{ Descrição da atitude } \\
\hline$p(\%)$ & $t(\mathrm{~h})$ & & \\
\hline$\geq 85$ & $\leq 2$ & confia na informação (alta utilidade) & $\begin{array}{l}\text { usuário utiliza a informação meteorológica para tomar } \\
\text { decisões e avalia os valores de ' } x \text { ' (variáveis) na seleção de } \\
\text { portfólios }\end{array}$ \\
\hline $85>p \geq 20$ & $2<t \leq 24$ & confiança parcial & $\begin{array}{l}\text { informação meteorológica tem uma utilidade parcial na } \\
\text { decisão, pois os parâmetros ' } p \text { ' e ' } t \text { ' penalizam e reduzem o } \\
\text { nível de confiança do usuário }\end{array}$ \\
\hline$p<20$ & $t>24$ & não confia na informação (baixa utilidade) & $\begin{array}{l}\text { usuário não utiliza a informação meteorológica para tomar } \\
\text { decisões, independente dos valores de ' } x \text { ' (variáveis) }\end{array}$ \\
\hline
\end{tabular}

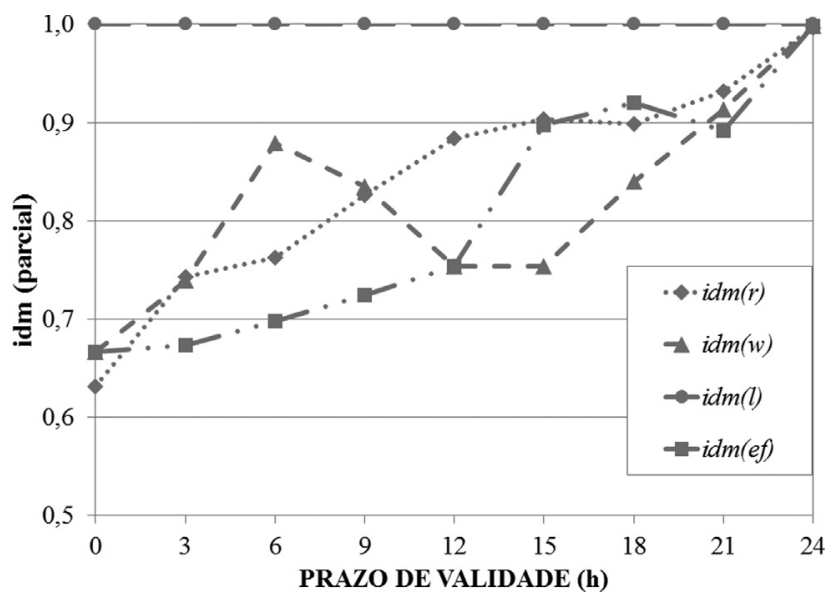

Figura 5 - Variação do idm parcial da AS para os quatro critérios meteorológicos.

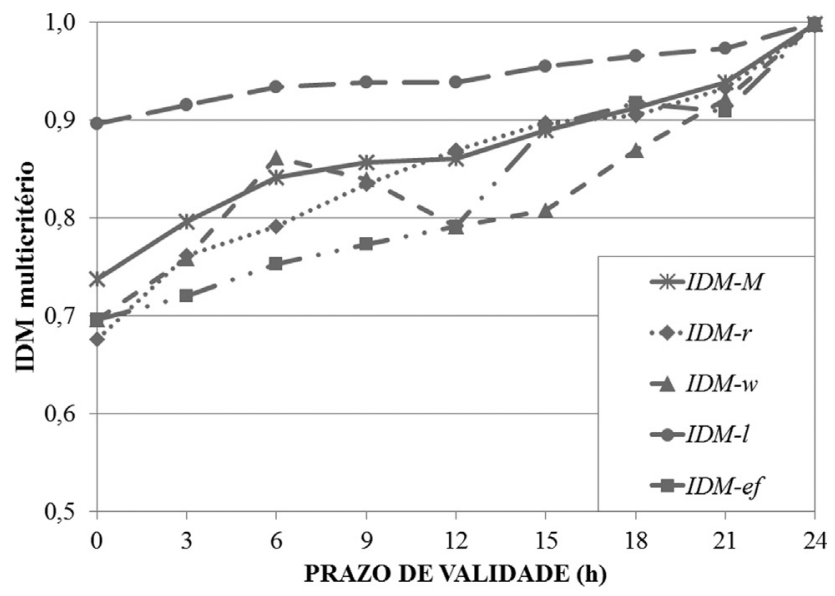

Figura 6 - Novos IDM globais da AS com a modificação dos pesos para os quatro critérios meteorológicos.

peso. Por exemplo, comparando as Figs. 5 e 6, a curva do idm parcial chuva (idm-r) tem a mesma tendência da curva $I D M-M$ (peso 0,35 ) e é praticamente a mesma para a curva $I D M-r$ (peso 0,7). Portanto, a AS sugere que uma vez estabelecido o cenário meteorológico, é fundamental identificar quais são as reais preferências do usuário em relação aos critérios. Em outras palavras, definir os valores dos pesos das variáveis meteorológicas de acordo com a lógica compensatória do modelo de decisão. Para detalhes dos valores utilizados na AS, ver o Apêndice 3 .

Em uma atividade operacional, tal como a missão de lançamento de foguetes, muitas vezes os decisores de alta responsabilidade não tem condições de avaliar todos os critérios meteorológicos. Neste sentido, uma das principais vantagens do modelo de apoio à decisão através de um único índice (Tabela 6) é adequar uma interface de visualização sem a necessidade de detalhar as condições atmosféricas ou mesmo de apresentar campos meteorológicos de difícil interpretação. Com os valores do IDM global o usuário pode facilmente identificar as melhores recomendações a serem adotadas com o propósito de minimizar os potenciais impactos de condições meteorológicas adversas durante todo o prazo de validade da informação.

\section{Considerações Finais}

Este artigo teve como objetivo apresentar uma abordagem de apoio à decisão em condições de incerteza meteorológica para a proteção das infraestruturas do Centro de Lançamento de Alcântara (CLA). Para isso foi desenvolvido o Índice de Decisão Meteorológico (IDM) que incorporou as preferências dos usuários não-meteorologistas em relação às três características relacionadas à informação meteorológica: probabilidades da previsão, prazos de validade e os valores das variáveis atmosféricas consideradas.

$\mathrm{Na}$ construção deste modelo de apoio à decisão foram utilizados os conceitos de Analise de Decisão por Multicritério através de uma abordagem inovadora e agrupando as características de um problema de decisão utilizando a previsão de tempo probabilística. Como estudo de caso, foi aplicado o modelo utilizando o IDM em um processo decisório para proteção do Veículo Lançador de Satélite e da Torre Móvel de Integração no CLA.

Os resultados demonstraram que a proposta do IDM conseguiu representar as atitudes do usuário em relação às incertezas da informação meteorológica. A abordagem quantitativa desenvolvida com um índice único incorporou a principal estrutura de preferência dos usuários do Programa Espacial Brasileiro em relação aos prognósticos me- 
teorológicos. Para probabilidades baixas $(<20 \%)$ ou para prazos de validade estendidos ( $>24 \mathrm{~h}$ ) o usuário não utiliza a previsão de tempo para tomar decisões, mesmo com o prognóstico de condições desfavoráveis extremas. Isso demonstrou que o usuário escolhe aguardar uma atualização da previsão de tempo de modo a maximizar a recompensa considerando as probabilidades, os prazos de validade e os valores dos critérios.

$\mathrm{O}$ apoio à decisão através de um índice também facilitou a visualização das recomendações em um processo decisório operacional rápido e dinâmico. Entretanto, é necessário ressaltar o que o valor do IDM não significa que o risco meteorológico aumenta ou diminui, ou mesmo que uma decisão é certa ou errada. A finalidade do IDM é modelar a atitude através das preferências dos usuários, de forma a identificar quando a informação meteorológica disponível é útil (ou não) dentro de um determinado contexto decisório. Como aplicações diretas destes resultados, os meteorologistas podem desenvolver novos produtos customizados para cada usuário, de acordo com as preferências. Exemplo: concentrar os esforços da modelagem numérica de tempo para o prazo máximo que o usuário utiliza - que neste estudo de caso foi de $24 \mathrm{~h}$ - ou criar mapas regionais bidimensionais ou tabelas com os prognósticos dos IDM baseados na previsão meteorológica georeferenciada.

Como comentário final, vale destacar que a integração das técnicas de previsão de tempo probabilística com métodos da Pesquisa Operacional, através de uma abordagem multi-metodológica pode ser utilizada com diversas configurações e em outras situações de decisão meteorológica em condições de incerteza.

\section{Agradecimentos}

Os autores agradecem o suporte financeiro do $\mathrm{CNPq}$ (Processo $\mathrm{n}^{\circ}$ 142212/2011-3 e 232898/2014-6) e da CAPES (Processo $\mathrm{n}^{\circ}$ 14552/2013- 02). Agradecimento também a todos os entrevistados do DCTA, IAE, CLBI, ACS e INPE pelos comentários e contribuições. No entanto, quaisquer opiniões, conclusões ou sugestões neste artigo são de responsabilidade dos autores e não refletem necessariamente os pontos de vista das instituições do Programa Espacial Brasileiro. Parte do conteúdo metodológico deste artigo é integrante de um depósito de pedido de patente internacional (Protocolo $\mathrm{n}^{\circ}$ PCT/BR2016/050232).

\section{Referências}

ACKERMANN, F.; EDEN, C. SODA - Journey Making and Mapping in Practice. In: ROSENHEAD, J.; MINGERS, J. (Eds.). . Rational Analysis for a Problematic World Revisited: Problem Structuring Methods for Complexity. 2. ed. West Sussex: Wiley, 2001. p. 43-60.

ALMEIDA, A.T. O Conhecimento e o Uso de Métodos Multicritério de Apoio a Decisão. 2. ed. Recife: Editora UFPE, 2011.
BELTON, V.; STEWART, T.J. Multiple Criteria Decision Analysis: An Integrated Approach. 1. ed. Berlin: Springer, 2002.

BRITO, A.J.M.; ALMEIDA, A.T. Modeling a multi-attribute utility newsvendor with partial backlogging. Eur J Oper Res, v. 220, n. 3, p. 820-830, 2012.

CAMPELLO-DE-SOUZA, F.M. Decisões Racionais em Situações de Incerteza. 2. ed. Recife: Vade Mecum, 2007.

CARUZZO, A.; BELDERRAIN, M.C.N.; FISCH, G.; MANSO, D.F. Mapping of the aerospace meteorology in the Brazilian Space Program: challenges and opportunities to rocket launch. Journal of Aerospace Technology and Management, v. 7, n. 1, p. 7-18, 2015.

CARUZZO, A.; ZAWADZKI, M.; BELDERRAIN, M.C.N. Proteção de Infraestruturas Críticas: desafios da previsão meteorológica como ferramenta de apoio aos Serviços de Inteligência. Revista Brasileira de Inteligência, n. 9, p. 51-63, 2015a.

CLEMEN, R. T. Making Hard Decisions: An Introduction to Decision Analysis. 2. ed. Boston: Duxbury Press, 1997.

DEMUTH, J.L.; MORSS, R.E.; MORROW, B.H.; LAZO, J.K. Creation and Communication of Hurricane Risk Information. B Am Meteorol Soc, v. 93, n. 8, p. 1133-1145, 2012.

DOLIF, G. et al. Resilience and brittleness in the ALERTA RIO system: a field study about the decision-making of forecasters. Nat Hazards, v. 65, n. 3, p. 1831-1847, 2013.

ENSSLIN, L. et al. Avaliação do desempenho de empresas terceirizadas com o uso da metodologia multicritério de apoio à decisão - construtivista. Pesquisa Operacional, v. 30, n. 1, p. 125-152, 2010.

ENSSLIN, L.; MONTIBELLER, G.; NORONHA, S.M. Apoio à Decisão - Metodologias para Estruturação de Problemas e Avaliação Multicritério de Alternativas. 2. ed. Florianópolis: Editora Insular, 2001.

FAN, F. M. et al. Sobre o uso da persistência de previsões determinísticas de vazão para a tomada de decisão. Rev. Bras. Meteorol., v. 31, n. 2, p. 218-228, 2016.

FAN, F.M.; RAMOS, M.H.; COLLISCHONN, W. Sobre o uso de previsões hidrológicas probabilísticas para tomada de decisão. Revista Brasileira de Recursos Hídricos, v. 20, n. 4, p. 914-926, 2015.

GEORGIOU, I. Cognitive Mapping and Strategic Options Development and Analysis (SODA). In: COCHRAN, J. J. (Ed.). . Wiley Encyclopedia of Operations Research and Management Science. [s.1.] John Wiley \& Sons, 2010. p. 1-10.

GOMES, L.F.A.M.; RANGEL, L.A.D.; LEAL-JUNIOR, M.R. Treatment of uncertainty through the interval Smart/Swing Weighting Method: a case study. Pesquisa Operacional, v. 31, n. 3, p. 467-485, 2011.

GUIKEMA, S.D.; QUIRING, S.M.; HAN, S.R. Prestorm estimation of hurricane damage to electric power distribution systems. Risk Anal, v. 30, n. 12, p. 1744-52, 2010.

HANLON, C.J. et al. Probabilistic forecasting for isolated thunderstorms using a genetic algorithm: The DC3 campaign. J. Geophys. Res. Atmos., v. 119, n. 1, p. 65-74, 2014.

HIRAMATSU, A.; HUYNH, V.N.; NAKAMORI, Y. A behavioral analysis in decision making using weather information with the fuzzy target. In: TORRA, V.; NARUKAWA, Y.; YOSHIDA, Y. (Eds.). . Modeling Decisions for Artifi- 
cial Intelligence. 1. ed. Kitakyushu: Springer, 2007. p. 6879.

JOSLYN, S.; LECLERC, J. Uncertainty forecasts improve weather-related decisions and attenuate the effects of forecast error. J Exp Psychol Appl, v. 18, n. 1, p. 126-140, 2012.

JOSLYN, S.; LECLERC, J. Decisions With Uncertainty: The Glass Half Full. Curr Dir Psychol Sci, v. 22, n. 4, p. 308-315, 2013.

JOSLYN, S.; SAVELLI, S.; NADAV-GREENBERG, L. Reducing probabilistic weather forecasts to the worst-case scenario: anchoring effects. J Exp Psychol-Appl, v. 17, n. 4, p. 342-353, 2011.

KEENEY, R.L.; RAIFFA, H. Decisions with Multiple Objectives: Preferences and Value Trade-Offs. 1. ed. New York: Cambridge University Press, 1993.

LI, H. et al. Ranking the risks from multiple hazards in a small community. Risk Anal, v. 29, n. 3, p. 438-456, 2009.

MACKENZIE, C.A. Summarizing Risk Using Risk Measures and Risk Indices. Risk Anal, v. 34, n. 12, p. 2143-2162, 2014.

MARQUES, R.F.C.; FISCH, G. As atividades de Meteorologia Aeroespacial no Centro Técnico Aeroespacial (CTA). Boletim da Sociedade Brasileira de Meteorologia, v. 29, n. 3, p. 23-27, 2005.

MONTIBELLER, G.; FRANCO, L.A. Decision and Risk Analysis for the evaluation of Strategic Options. In: O'BRIEN, F. A.; DYSON, R. G. (Eds.). Supporting strategy: frameworks, methods and models. 1 ed. Chichester: Wiley, 2007. p. 251-284.

NEAL, R.A. et al. Ensemble based first guess support towards a risk-based severe weather warning service. Meteorol Appl, v. 21, n. 3, p. 563-577, 2014.

OH, E.H.; DESHMUKH, A.; HASTAK, M. Disaster impact analysis based on inter-relationship of critical infrastructure and associated industries: A winter flood disaster event. International Journal of Disaster Resilience in the Built Environment, v. 1, n. 1, p. 25-49, 2010.

QUIRING, S.M.; SCHUMACHER, A.B.; GUIKEMA, S.D. Incorporating Hurricane Forecast Uncertainty into a Decision Support Application for Power Outage Modeling. B. Am. Meteorol. Soc., v. 95, n. 1, p. 47-58, 2014.

REGNIER, E. Public Evacuation Decisions and Hurricane Track Uncertainty. Manage Sci, v. 54, n. 1, p. 16-28, 2008.

SCHAD, I. et al. Why do people not learn from flood disasters? Evidence from Vietnam's northwestern mountains. Nat Hazards, v. 62, n. 2, p. 221-241, 2012.

SILVA, A.F.G.; FISCH, G. Avaliação do modelo WRF para a previsão do perfil do vento no Centro de Lançamento de Alcântara. Rev. Bras. Meteorol., v. 29, n. 2, p. 259-270, 2014.

SLINGO, J.; PALMER, T. Uncertainty in weather and climate prediction. Phil. Trans. R. Soc., v. 369, n. 1956, p. 4751-67, 2011.

VAUGHAN, D. The Challenger Launch Decision: Risky Technology, Culture, and Deviance at NASA. 1 ed. Chicago: University of Chicago Press, 1996.

VAUGHAN, W.W.; JOHNSON, D.L. Aerospace meteorology: An overview of some key environmental elements. J. Aerosp. Technol. Manag., v. 5, n. 1, p. 7-14, 2013.

WILSON, L.J.; GILES, A. A new index for the verification of accuracy and timeliness of weather warnings. Meteorol Appl, v. 20, n. 2, p. 206-216, 2013.

This is an Open Access article distributed under the terms of the Creative Commons Attribution Non-Commercial License which permits unrestricted non-commercial use, distribution, and reproduction in any medium provided the original work is properly cited. 


\section{Apêndice 1}

Função de valor parcial para critério precipitação $\left(\mathrm{mm} \mathrm{h}^{-1}\right)$

$$
I(r)=\left\{\begin{array}{r}
1, r \leq 1 \\
-\left(\left(\frac{r}{19}\right)-1,053\right), 1<r \leq 20 \\
0, r>20
\end{array}\right.
$$

Função de valor parcial para o critério vento em superfície $\left(\mathrm{m} \mathrm{s}^{-1}\right)$

$$
I(w)=\left\{\begin{array}{r}
1, w \leq 10 \\
-\left(\left(\frac{w}{30}\right)-1,333\right), 10<w \leq 40 \\
0, w>40
\end{array}\right.
$$

Função de valor parcial para o critério ocorrência de relâmpago (km)

$$
I(l)=\left\{\begin{array}{r}
1, l \geq 50 \\
\left(\frac{l}{40}\right)-0,25,10 \leq l<50 \\
0, l<10
\end{array}\right.
$$

Função de valor parcial para valor do campo elétrico no CLA $\left(\mathrm{kV} \mathrm{m}^{-1}\right)$

$$
1, \text { ef } \leq 0,6
$$

$$
I(e f)=\{-((1,667 e f)-2), 0,6<\text { ef } \leq 1,2
$$

0, ef $>1,2$ 


\section{Apêndice 2}

Valores utilizados para as três condições hipotéticas de previsão de tempo para o estudo de caso

\begin{tabular}{lcccc}
\hline & Chuva & Vento & Relamp & Campo elétrico \\
\hline Peso entre critérios & 0,35 & 0,25 & 0,25 & 0,15 \\
\hline
\end{tabular}

\begin{tabular}{lcccccccccc}
\hline \multicolumn{7}{c}{ Parâmetros da informação meteorológica (observação + previsão de tempo) } \\
\hline prazo de validade (h) & 0 & 3 & 6 & 9 & 12 & 15 & 18 & 21 & 24 \\
probabilidade (\%) & 100 & 100 & 95 & 90 & 85 & 80 & 75 & 70 & 70 \\
$I(t)$ & 1 & 0,9546 & 0,8183 & 0,6819 & 0,5455 & 0,4092 & 0,2728 & 0,1365 & 0,0001 \\
$I(p)$ & 1 & 1 & 1 & 1 & 1 & 0,9228 & 0,8458 & 0,7689 & 0,7689 \\
\hline
\end{tabular}

\begin{tabular}{lccccccccc}
\hline & \multicolumn{1}{c}{ Condição Meteorológica - Otimista $(\mathrm{O})$} & & \\
\hline prazo de validade $(\mathrm{h})$ & 0 & 3 & 6 & 9 & 12 & 15 & 18 & 21 & 24 \\
probabilidade $(\%)$ & 100 & 100 & 95 & 90 & 85 & 80 & 75 & 70 & 70 \\
chuva $\left(\mathrm{mm} \mathrm{h}^{-1}\right)$ & 0 & 0 & 0 & 0 & 0 & 0 & 0 & 0 & 0 \\
vento $\left(\mathrm{m} \mathrm{s}^{-1}\right)$ & 24 & 16 & 12 & 10 & 9 & 8 & 8 & 7 & 6 \\
relâmpago $(\mathrm{km})$ & 100 & 100 & 100 & 100 & 100 & 100 & 100 & 100 & 100 \\
campo elétrico $\left(\mathrm{kV} \mathrm{m}^{-1}\right)$ & 0,2 & 0,3 & 0,0 & 0,2 & 0,2 & 0,3 & 0,2 & 0,1 & 0,2 \\
\hline
\end{tabular}

\begin{tabular}{|c|c|c|c|c|c|c|c|c|c|}
\hline \multicolumn{10}{|c|}{ Índice 'n’ (Otimista) } \\
\hline $\mathrm{I}(\mathrm{r})$ & 1 & 1 & 1 & 1 & 1 & 1 & 1 & 1 & 1 \\
\hline $\mathrm{I}(\mathrm{w})$ & 0,5330 & 0,7997 & 0,9330 & 1 & 1 & 1 & 1 & 1 & 1 \\
\hline I(l) & 1 & 1 & 1 & 1 & 1 & 1 & 1 & 1 & 1 \\
\hline I(ef) & 1 & 1 & 1 & 1 & 1 & 1 & 1 & 1 & 1 \\
\hline \multicolumn{10}{|c|}{ idm parcial } \\
\hline $\operatorname{idm}(r)$ & 1 & 1 & 1 & 1 & 1 & 1 & 1 & 1 & 1 \\
\hline $\operatorname{idm}(w)$ & 0,5330 & 0,8043 & 0,9394 & 1 & 1 & 1 & 1 & 1 & 1 \\
\hline $\operatorname{idm}(1)$ & 1 & 1 & 1 & 1 & 1 & 1 & 1 & 1 & 1 \\
\hline $\mathrm{idm}(\mathrm{ef})$ & 1 & 1 & 1 & 1 & 1 & 1 & 1 & 1 & 1 \\
\hline IDM-O & 0,8833 & 0,9511 & 0,9848 & 1,0000 & 1,0000 & 1,0000 & 1,0000 & 1,0000 & 1,0000 \\
\hline Portfolio & M2 & M2 & M2 & M1 & M1 & M1 & M1 & M1 & M1 \\
\hline
\end{tabular}

\begin{tabular}{lccccccccc}
\hline & \multicolumn{1}{c}{ Condição Meteorológica - Intermediária (M) } \\
\hline prazo de validade (h) & 0 & 3 & 6 & 9 & 12 & 15 & 18 & 21 & 24 \\
probabilidade (\%) & 100 & 100 & 95 & 90 & 85 & 80 & 75 & 70 & 70 \\
chuva $\left(\mathrm{mm} \mathrm{h}^{-1}\right)$ & 8 & 6 & 6 & 5 & 4 & 4 & 5 & 5 & 10 \\
vento $\left(\mathrm{m} \mathrm{s}^{-1}\right)$ & 20 & 18 & 14 & 16 & 20 & 22 & 20 & 18 & 15 \\
relâmpago $\left(\mathrm{km}^{-1}\right)$ & 95 & 80 & 80 & 75 & 70 & 71 & 60 & 55 & 55 \\
campo elétrico $\left(\mathrm{kV} \mathrm{m}^{-1}\right)$ & 0,8 & 0,8 & 0,8 & 0,8 & 0,8 & 0,7 & 0,7 & 0,8 & 0,7 \\
\hline
\end{tabular}




\begin{tabular}{lccccccccc}
\hline \multicolumn{1}{c}{ Índice ' $n$ ' (Intermediária) } \\
\hline I(r) & 0,6319 & 0,7372 & 0,7372 & 0,7898 & 0,8425 & 0,8425 & 0,7898 & 0,7898 & 0,5267 \\
I(w) & 0,6663 & 0,7330 & 0,8663 & 0,7997 & 0,6663 & 0,5997 & 0,6663 & 0,7330 & 0,8330 \\
I(l) & 1 & 1 & 1 & 1 & 1 & 1 & 1 & 1 & 1 \\
I(ef) & 0,6664 & 0,6664 & 0,6664 & 0,6664 & 0,6664 & 0,8331 & 0,8331 & 0,6664 & 0,8331 \\
\hline idm parcial & & & & & & & & \\
\hline idm(r) & 0,6319 & 0,7432 & 0,7623 & 0,8265 & 0,8836 & 0,9032 & 0,8990 & 0,9319 & 0,9960 \\
idm(w) & 0,6663 & 0,7391 & 0,8791 & 0,8346 & 0,7536 & 0,7540 & 0,8397 & 0,9135 & 0,9986 \\
idm(1) & 1 & 1 & 1 & 1 & 1 & 1 & 1 & 1 \\
idm(ef) & 0,6664 & 0,6741 & 0,6982 & 0,7245 & 0,7536 & 0,8974 & 0,9198 & 0,8919 & 0,9986 \\
IDM-M & 0,7377 & 0,7960 & 0,8413 & 0,8566 & 0,8607 & 0,8892 & 0,9126 & 0,9383 & 0,9981 \\
Portfolio & M3 & M3 & M3 & M2 & M2 & M2 & M2 & M2 & M2 \\
\hline & & & & & & & & &
\end{tabular}

\begin{tabular}{|c|c|c|c|c|c|c|c|c|c|}
\hline \multicolumn{10}{|c|}{ Condição Meteorológica - Pessimista (P) } \\
\hline prazo de validade (h) & 0 & 3 & 6 & 9 & 12 & 15 & 18 & 21 & 24 \\
\hline probabilidade $(\%)$ & 100 & 100 & 95 & 90 & 85 & 80 & 75 & 70 & 70 \\
\hline chuva $\left(\mathrm{mm} \mathrm{h}^{-1}\right)$ & 10 & 8 & 6 & 10 & 7 & 5 & 5 & 5 & 10 \\
\hline vento $\left(\mathrm{m} \mathrm{s}^{-1}\right)$ & 14 & 15 & 15 & 15 & 22 & 22 & 20 & 18 & 15 \\
\hline relâmpago $(\mathrm{km})$ & 45 & 40 & 30 & 25 & 15 & 10 & 8 & 5 & 0 \\
\hline campo elétrico $\left(\mathrm{kV} \mathrm{m}^{-1}\right)$ & 0,7 & 0,8 & 0,9 & 0,8 & 0,9 & 1,0 & 1,2 & 1,4 & 1,8 \\
\hline \multicolumn{10}{|c|}{ Índice ' $n$ ' (Pessimista) } \\
\hline $\mathrm{I}(\mathrm{r})$ & 0,5267 & 0,6319 & 0,7372 & 0,5267 & 0,6846 & 0,7898 & 0,7898 & 0,7898 & 0,5267 \\
\hline $\mathrm{I}(\mathrm{w})$ & 0,8663 & 0,8330 & 0,8330 & 0,8330 & 0,5997 & 0,5997 & 0,6663 & 0,7330 & 0,8330 \\
\hline $\mathrm{I}(1)$ & 0,8750 & 0,7500 & 0,5000 & 0,3750 & 0,1250 & 0 & 0 & 0 & 0 \\
\hline $\mathrm{I}(\mathrm{ef})$ & 0,8331 & 0,6664 & 0,4997 & 0,6664 & 0,4997 & 0,3330 & 0 & 0 & 0 \\
\hline \multicolumn{10}{|l|}{ idm parcial } \\
\hline $\operatorname{idm}(r)$ & 0,5267 & 0,6404 & 0,7623 & 0,6091 & 0,7670 & 0,8709 & 0,8990 & 0,9319 & 0,9960 \\
\hline $\operatorname{idm}(w)$ & 0,8663 & 0,8368 & 0,8489 & 0,8621 & 0,7043 & 0,7540 & 0,8397 & 0,9135 & 0,9986 \\
\hline $\operatorname{idm}(1)$ & 0,8750 & 0,7557 & 0,5477 & 0,4839 & 0,3537 & 0,3855 & 0,5196 & 0,6761 & 0,9916 \\
\hline $\operatorname{idm}(e f)$ & 0,8331 & 0,6741 & 0,5474 & 0,7245 & 0,6305 & 0,5901 & 0,5194 & 0,6761 & 0,9916 \\
\hline IDM-P & 0,7446 & 0,7234 & 0,6981 & 0,6584 & 0,6275 & 0,6782 & 0,7324 & 0,8250 & 0,9949 \\
\hline Portfolio & M3 & M3 & M3 & M4 & M4 & M3 & M3 & M3 & M2 \\
\hline
\end{tabular}




\section{Apêndice 3}

Valores utilizados na Análise de Sensibilidade do estudo de caso

\begin{tabular}{lccccc}
\hline & & & & \\
& & & & \\
Critério meteorológico & IDM-M & IDM-r & IDM-w & IDM-1 & IDM-ef \\
\hline chuva & 0,35 & 0,70 & 0,10 & 0,10 & 0,10 \\
vento & 0,25 & 0,10 & 0,70 & 0,10 & 0,10 \\
relâmpago & 0,25 & 0,10 & 0,10 & 0,70 & 0,10 \\
campo elétrico & 0,15 & 0,10 & 0,10 & 0,10 & 0,70 \\
\hline
\end{tabular}

\begin{tabular}{lcccccccccc}
\hline \multicolumn{7}{c}{ Parâmetros da informação meteorológica (observação + previsão de tempo) } \\
\hline prazo de validade (h) & 0 & 3 & 6 & 9 & 12 & 15 & 18 & 21 & 24 \\
probabilidade (\%) & 100 & 100 & 95 & 90 & 85 & 80 & 75 & 70 & 70 \\
$I(t)$ & 1 & 0,9546 & 0,8183 & 0,6819 & 0,5455 & 0,4092 & 0,2728 & 0,1365 & 0,0001 \\
$I(p)$ & 1 & 1 & 1 & 1 & 1 & 0,9228 & 0,8458 & 0,7689 & 0,7689 \\
\hline
\end{tabular}

\begin{tabular}{|c|c|c|c|c|c|c|c|c|c|}
\hline \multicolumn{10}{|c|}{ Condição Meteorológica - Intermediária (M) } \\
\hline chuva $\left(\mathrm{mm} \mathrm{h}^{-1}\right)$ & 8 & 6 & 6 & 5 & 4 & 4 & 5 & 5 & 10 \\
\hline vento $\left(\mathrm{m} \mathrm{s}^{-1}\right)$ & 20 & 18 & 14 & 16 & 20 & 22 & 20 & 18 & 15 \\
\hline relâmpago (km) & 95 & 80 & 80 & 75 & 70 & 71 & 60 & 55 & 55 \\
\hline campo elétrico $\left(\mathrm{kV} \mathrm{m}^{-1}\right)$ & 0,8 & 0,8 & 0,8 & 0,8 & 0,8 & 0,7 & 0,7 & 0,8 & 0,7 \\
\hline \multicolumn{10}{|c|}{ Índice ‘n’ (Intermediária) } \\
\hline$I(r)$ & 0,6319 & 0,7372 & 0,7372 & 0,7898 & 0,8425 & 0,8425 & 0,7898 & 0,7898 & 0,5267 \\
\hline$I(w)$ & 0,6663 & 0,7330 & 0,8663 & 0,7997 & 0,6663 & 0,5997 & 0,6663 & 0,7330 & 0,8330 \\
\hline$I(l)$ & 1 & 1 & 1 & 1 & 1 & 1 & 1 & 1 & 1 \\
\hline$I(e f)$ & 0,6664 & 0,6664 & 0,6664 & 0,6664 & 0,6664 & 0,8331 & 0,8331 & 0,6664 & 0,8331 \\
\hline \multicolumn{10}{|l|}{ idm parcial } \\
\hline $\operatorname{idm}(r)$ & 0,6319 & 0,7432 & 0,7623 & 0,8265 & 0,8836 & 0,9032 & 0,8990 & 0,9319 & 0,9960 \\
\hline $\operatorname{idm}(w)$ & 0,6663 & 0,7391 & 0,8791 & 0,8346 & 0,7536 & 0,7540 & 0,8397 & 0,9135 & 0,9986 \\
\hline $\operatorname{idm}(l)$ & 1 & 1 & 1 & 1 & 1 & 1 & 1 & 1 & 1 \\
\hline $\operatorname{idm}(e f)$ & 0,6664 & 0,6741 & 0,6982 & 0,7245 & 0,7536 & 0,8974 & 0,9198 & 0,8919 & 0,9986 \\
\hline \multicolumn{10}{|l|}{ IDM Global } \\
\hline IDM-M & 0,7377 & 0,7960 & 0,8413 & 0,8566 & 0,8607 & 0,8892 & 0,9126 & 0,9383 & 0,9981 \\
\hline IDM-r & 0,6756 & 0,7616 & 0,7913 & 0,8344 & 0,8693 & 0,8974 & 0,9053 & 0,9329 & 0,9970 \\
\hline IDM-w & 0,6963 & 0,7591 & 0,8614 & 0,8393 & 0,7912 & 0,8079 & 0,8697 & 0,9218 & 0,9985 \\
\hline IDM-1 & 0,8965 & 0,9156 & 0,9340 & 0,9386 & 0,9391 & 0,9555 & 0,9659 & 0,9737 & 0,9993 \\
\hline IDM-ef & 0,6963 & 0,7201 & 0,7529 & 0,7733 & 0,7912 & 0,8939 & 0,9178 & 0,9089 & 0,9985 \\
\hline
\end{tabular}

